ten Verfall in Strafverfahren, die letztlich ausländerrechtliche Ziele verfolgen.

\section{Fußnoten:}

1 So auch Christian Pfeiffer, Kriminalistik 2005, 217, allerdings mit anderen Berechnungen.

2 Wer nichts gegen den weiten Gewaltbegriff von Ursula Müller in der vom BMFSFJ am 24.09.2004 vorgestellten Gewaltstudie hat, kann im Internet in der Zusammenfassung (S.24) nachlesen, dass Prostituierte und inhaftierte Frauen mit $92 \%$ gegenüber $58 \%$ (Hauptstudie) durchgängig angeben, in ihrem Leben bereits sexuell belästigt worden zu sein. Auch die Gewalterfahrung ist deutlich höher gegenüber der übrigen weiblichen Bevölkerung, was wenig verwunderlich ist, aber angesichts der erheblichen Differenz dennoch aussagekräftig ist.

Wer es genauer wissen will, wird zwischen jungen und älteren Prostituierten unterscheiden und erkennen, dass insbesondere die Jugendlichen auf dem Straßenstrich viel Brachialgewalt erleben, während erwachsene Prostituierte in entsprechenden Wohnungen ihr Leben sehr viel geregelter organisieren können, vgl. Leopold/Grieger, Das Parlament vom 20.12.2004 B 52 - 53/2004. Ein entsprechendes Forschungsvorhaben von Kavemann/Leopold, von den GRUNEN in Auftrag gegeben, geht dem noch genauer nach.

3 »Das war doch widerlich und gehört bestraft « kommentierte Siegfried Kauder und initiierte einen CDU-
Gesetzesentwurf zur sog. Freierbestrafung, SZ vom 25.01.2005.

4 Nur dann können sie eine befristete Aufenthaltserlaubnis erhalten.

5 Fischer, Tröndle/Fischer $\S 180$ a Rdnr. 27 überträgt diese Rechtsprechung neuerdings auch auf die ausbeuterische Prostitution und Zuhälterei, vgl. ferner § 291 Rdnr. 17. Die Kommentierung in diesem praktisch bedeutsamen Kommentar ist seit der 52 . Aufl. 2004 ausgesprochen offen für derartige Probleme, was zeigt, dass die Rechtsprechung eher fortschrittlicher ist als die oft populistische Gesetzgebung.

6 Beate Leopold/Elfriede Steffan, Evaluierung unterstützender Maßnahmen aus der Prostitution (EVAProjekt). SPI-Forschungs-GmbH, Berlin 1997.

\title{
Einwände gegen den am 19.02.2005 neu gefassten Straftatbestand des Menschenhandels in $\S 232$ Abs. 1 StGB
}

\author{
Monika Frommel und Martin Schaar
}

W er den an die Stelle des alten $\S 180 \mathrm{~b}$ StGB getretenen Auffangtatbestand des Menschenhandels liest, erkennt unschwer, dass hier weder ein Gewalt-, noch ein Täuschungsdelikt geschaffen worden ist, auch keine Spezialvorschrift zur Willensbeugung (wie Thoma in diesem Heft unterstellt), sondern eine Strafnorm, welche illegale Märkte kontrollieren will. Illegal sind diese Märkte aber in erster Linie wegen des Ausländerrechts. Kritisiert werden soll im folgenden nicht der gesamte Komplex der $\S \S 180$ b, 181 des alten und der seit 2005 in den $\S \S 232 \mathrm{ff}$ neu gefassten Tatbestände, sondern nur ein Ausschnitt: die Vermittlung von Nicht-EUBürgern und/oder jungen Frauen unter 21 Jahren in die Prostitution. Unberücksichtig bleiben hier also die $\S \S 232$ Abs. 4, 233 ff. StGB, deren Unrecht bis zum 19.02. 2005 im Verbrechenstatbestand des § 181 StGB (schwerer Menschenhandel) geregelt und nun lediglich mit Qualifikationen versehen worden sind, welche einerseits eine härtere, andererseits aber wegen der vorgesehenen minderschweren Fällen eine milde Bestrafung, sogar eine Informalisierung ermöglichen.

\section{$\S 232$ StGB (neu gefasst am 19.02.2005):}

(1) Wer eine andere Person unter Ausnutzung einer Zwangslage oder der Hilflosigkeit, die mit ihrem Aufenthalt in einem fremden Land verbunden ist, zur Aufnahme oder Fortsetzung der Prostitution oder dazu bringt, sexuelle Handlungen, durch die sie ausgebeutet wird, an oder vor dem Täter oder einem Dritten vor oder von dem Täter oder einem Dritten an sich vornehmen zu lassen, wird mit Freiheitsstrafe von sechs Monaten bis zu zehn Jahren bestraft. Ebenso wird bestraft, wer eine Person unter einundzwanzig Jahren zur Aufnahme oder Fortsetzung der Prostitution oder zu den sonst in Satz 1 bezeichneten sexuellen Handlungen bringt.

(2) Der Versuch ist strafbar.

Behandelt wird hier nur dieser Auffangtatbestand, der von der Gesetzgebung bewusst so weit wie möglich gefasst worden ist und jede Vermittlung von ausländischen und jungen Frauen und Männern unter 21 Jahren in die Prostitution bestraft. Ausübung von Zwang oder Täuschung ist hier - im Unterschied zu Abs. 4 nicht notwendig. Verfolgt wird also nicht nur die sog. Zwangsprostitution, sondern fast jede grenzüberschreitende Prostitutionsvermittlung. Setzt der Täter Gewalt, Drohung und List ein, dann wird er nach $\S 232$ Abs. 4 StGB erheblich härter bestraft (vgl. den Gesetzestext anbei). Zwar bestimmt der moralisch aufgeladene Begriff der Zwangsprostitution das Thema Menschenhandel, aber diese Assoziation ist vorschnell und irreführend. Kritisiert wird hier also nicht der in $\S 232$ Abs. 4 StGB normierte Verbrechenstatbestand des schweren Menschenhandels, der klare Täter-Opfer-Situationen und klare Tathandlungen beinhaltet (Gewalt, Drohung und List), schließlich wird die Ausübung von Zwang auch sonst strafrechtlich verboten, etwa als Erpressung, und Täuschungen kennen wir auch, allerdings überrascht die weite Tathandlung der List. Derartiges würde für Betrug nicht genügen. Auch haben wir es im Unter- schied zur ebenfalls sehr weit gefassten sexuellen Nötigung (vgl. die Urteilsanmerkung zu § 177 Abs. 1 Nr. 3 StGB - sexuelle Nötigung unter Ausnutzung einer schutzlosen Lage - in diesem Heft) beim oben zitierten Auffangtatbestand nicht mit einer individualisierten Täter-OpferBeziehung zu tun, sondern mit dem problematischen Versuch, jede Vermittlung in schwarze und graue Märkte zu sanktionieren und bei Gewerbsmäßigkeit sogar als Verbrechen (§ 232 Abs. 3 Nr. 3 StGB). Geschützt werden soll durch $\S 232$ StGB nicht das sexuelle Selbstbestimmungsrecht (dies war auch in $\S 180 \mathrm{~b}$ StGB nicht das Rechtsgut), sondern die wirtschaftliche und soziale Bewegungsfreiheit von Menschen, die in einer oft erst durch das Ausländerrecht geschaffenen Notlage und/oder einer unabhängig davon existierenden wirtschaftlichen Zwangslage » zu fast allem bereit« sind. Außerdem werden heranwachsende Prostituierte wie Jugendliche behandelt, also weitgehend von der Legalisierung sexueller Dienstleistungen ausgenommen.

Die Struktur eines solchen Tatbestandes unterscheidet sich erheblich von der des lange umstrittenen § 177 Abs. 1 Nr. 3 StGB (der sexuellen Nötigung unter Ausnutzung einer schutzlosen Lage), aber es gibt Gemeinsamkeiten. Immerhin benutzt die Gesetzgebung auch hier den Begriff des »Ausnutzens « einer Lage, die entweder mit einer erhöhten Schutzlosigkeit oder wie in § 232 mit einer Unfreiheit verbunden ist. Ähnlichkeiten bestehen auch zu der im übrigen so gut wie nicht praktisch umgesetzten Jugendschutznorm 
des $§ 182$ Abs. 1 Nr. 1 StGB. Gemeinsam sind diesen Tatbeständen diffuse Zwangslagen. Der Zwang ist hier ein sozialstruktureller. Allgemeine wirtschaftliche Bedrängnis und fehlende Sprachkenntnisse, ggf. auch die Angst vor der drohenden Abschiebung bei Menschen ohne Aufenthaltsbestimmung (der klassische Fall einer "auslandsspezifischen Hilflosigkeit", der zudem deutlich macht, dass wir es der Sache nach mit verkapptem Auslanderrecht zu tun haben). Erfasst werden bei dieser Opfergruppe typische Situationen fehlender oder verminderter Entscheidungsfreiheit.

Zu dieser Struktur des Tatbestandes passt auch der Verweis in der 2. Alternative (sexuelle Handlungen, durch die sie ausgebeutet werden) auf das Verbot der Ausbeutung von Prostituierten (geregelt in $\S \S 180$ a und 181 a StGB). Aber schon der Wortlaut des 2002 neu gefassten $§ 180$ a Abs. 1 StGB ist unklar. Verboten sind danach nicht schon - in Anlehnung an den Wuchertatbestand - unfaire Arbeitsbedingungen, sondern nach der Begründung zu diesem Gesetz, welche die Rechtsprechung strikt beachtet, nur solche, welche die Prostituierte in einer persönlichen oder wirtschaftlichen Abhängigkeit zu halten geeignet sind. Damit sind die Maßstäbe sehr hoch angesetzt. Außerdem rechtfertigt die Einwilligung der Prostituierten in überhöhte Preise, etwa das zu teuer vermietete Zimmer (es sei denn die Einwilligung ist unwirksam, weil sie »abhängigkeitsbedingt« ist). Die Gesetzgebung hat also die Chance vertan, diese hohen Hürden zu relativieren. Sie hat sie nun eher gefestigt als gelockert $^{1}$. Zwar ist es hM, dass es auf die persönliche Abhängigkeit der Prostituierten ankomme, mit der Folge dass eine strafrechtliche Kontrolle leer läuft, aber eine Begründung hierfür fehlt.

Sinnvoll wäre es eine Korrektur der geschlossenen Verträge bzw. der faktischen Arbeitsverhältnisse durch Zivil-, Miet- und Arbeitsrecht anzustreben und sich die dort gegebene Orientierung an $\S 242$ BGB zunutze zu machen (wieso dies nicht geschieht, Frommel, in diesem Heft). Aber dann müsste man Zivilverfahren anstrengen; denn strafrechtsdogmatisch kommt man nicht weiter, weil fast in allen Fällen zu hohe Preise durch die Einwilligung der Prostituierten gerechtfertigt wären. Es ist also ziemlich unrealistisch den Markt für sexuelle Dienstleistungen strafrechtlich regulieren zu wollen. Selbst Marx hätte sich 1867 gescheut, einen Tatbestand: »Ausbeutung ist verboten!« als Lösung für einen unkontrollierten Kapitalismus $\mathrm{zu}$ empfehlen. Aber im Ergebnis wird § 232 StGB damit zu einem ziemlich sinnlosen Straftatbestand, wenn man den Betroffenen damit helfen will.

- Die eigentliche Tathandlung, das Zuführen (»bringen «) erwachsener Personen zu einem allenfalls anrüchigen Gewerbe ist ein legales Verhalten, ist kein »Unrecht«.

- Bestraft werden soll dennoch, wer entweder selbst vermittelt oder aber die Dienstleistun- gen organisiert, wenn er oder sie dabei die Zwangslage, auslandsspezifische Hilflosigkeit und/ oder die jugendspezifische (ein noch unbestimmterer Begriff) Hilflosigkeit der freiwillig und ohne Täuschung dort tätigen Prostituierten ausnutzt.

- Das »Ausnutzen « der Zwangslage lässt sich nicht als Tathandlung fassen, da diese schließlich vorgefunden wird.

- Grundsätzlich muss ein verbotenes und vom Täter angestrebtes Handlungsziel entweder selbst eine Straftat sein, hier wäre etwa an ausbeuterische Prostitution oder ausbeuterische Zuhälterei nach $\S \S 180 \mathrm{a}, 181 \mathrm{a}$ StGB zu denken. Dann ließe sich das Unrecht als selbstständig vertypte Beihilfe zu diesen Delikten definieren. $\S 232$ Abs. 1 StGB ist aber nicht so konstruiert. Da eine "Ausbeutung " der vermittelten erwachsenen Prostituierten nicht erforderlich ist. Die in ihrer Entscheidung eingeschränkten Personen müssen nicht in einen solchen Bordellbetrieb (im weitesten Sinne) vermittelt werden, für den ein grobes Missverhältnis zwischen Leistung und Entgelt charakteristisch ist, Wucher nach § 291 StGB, sondern schlicht zur Prostitution, d.h. einem unter jedem denkbaren Gesichtspunkt legalen Verhalten.

- Nicht ausbeuterische Prostitution, sondern jede grenzüberschreitende Vermittlung und Beschäftigung soll demnach als organisierte Kriminalität bestraft werden. Da ein derartiges Anliegen aber ausschließlich eine Sache des ebenfalls notorisch umstrittenen Ausländerrechts ${ }^{2}$ ist, werden beim Thema "Menschenhandel und Zwangsprostitution " gezielt emanzipativ klingende Floskeln in einen ausländerrechtlichen Kontext eingestreut, um publikumswirksam jeden $>$ Handel mit der Ware Mensch anzuprangern. Der Sache nach soll aber nur der >Handel mit Nicht-EUBürger erschwert werden; denn nur diese sind typischerweise ohne Aufenthaltserlaubnis tätig und deshalb in einer »auslandsspezifischen Hilflosigkeit« gefangen. Nur bei ihnen kann die Kontrolle des Ausweises ein Strafverfahren nach sich ziehen, in dessen Verlauf es für die Beschuldigten interessant werden kann, als Zeuginnen aufzutreten.

Auf der strafrechtlichen Arena werden also bewusst Wertungswidersprüche zum ProstG hingenommen und die im Zivilrecht 2002 getroffenen Entscheidungen ignoriert. Danach ist nun einmal Prostitution als solche nicht verboten, so dass es auch wenig gute Gründe gibt zur Verfolgung derer, die lediglich die freiwillige Prostituion Erwachsener organisieren. Dennoch ermittelt die Polizei und überlässt es StA und Rechtsprechung, ob angeklagt wird und wie für den Fall, dass es zu einer Anklage gekommen ist, angemessene Konturen herausbildet. Auf derartige Zumutungen kann man kaum angemessen reagieren. Schon bei den nicht minder unbestimmten $\$ \S 180 \mathrm{a}$, 181 a StGB und den bis Februar 2005 geltenden
$\S \S 180 \mathrm{~b}, 181$ StGB überlegten sich Staatsanwaltschaften sehr genau, ob sie anklagen oder nicht, weil sie wissen, dass die Gerichte hohe Anforderungen an den Tatnachweis dieser Absichtsdelikte gestellt haben. Das bis Februar geltende Strafrecht zum Frauenhandel war nicht objektivierbar, aber auch der seit dem Februar 2005 geltende Grundtatbestand des § 232 kollidiert mit dem ProstG und kann gar nicht so umgesetzt werden wie es der Wortlaut nahe legt. Das alte wie das neue Recht erschöpfen sich mehr oder weniger in der Missbilligung des Erwerbstriebs der »Frauenhändler «. Die Probleme der Betroffenen könnten durch Straftatbestände wie Wucher und Erpressung sehr viel besser gelöst werden. Vermutlich wird es deshalb bei der Strategie der Vermeidung einer gerichtlichen Überprüfung bleiben. Fragen wir deshalb, wie wir selbst in der Richterrolle verfahren würden:

Ungenaues Strafrecht, das überdies Straftatbestände der Organisierten Kriminalität betrifft, schafft in erster Linie Ermittlungsparagrafen. Die Polizei erhält Befugnisse zur Beobachtung von Szenen, die ihr dubios erscheinen ${ }^{3}$ und die sie deshalb überwacht ${ }^{4}$. Bei den sog. "Schlepper, Schleußer, Frauen- und Menschenhändler « kommt noch hinzu, dass der Zweck der Beobachtung in erster Linie der ist, die Grenzen (gemeint sind beim Thema Frauenhandel zur Zeit die Grenzen zu den Staaten der erweiterten EU im Osten und zu den noch nicht oder auch künftig nicht der EU beitretenden Ländern Osteuropas) nur so weit zu öffnen, wie es nun einmal unumgänglich ist. Es kommt also viel zusammen bei dieser Art der Prävention.

\section{Wann und wie kann man ungenaues Strafrecht präzisieren?}

Die Methode ist immer dieselbe. Man fragt, was die Gesetzgebung bezweckt hat, ob dies legitim ist oder ob sie dabei über das Ziel hinausgeschossen ist und überlegt dann, welche Worte restriktiv ausgelegt werden müssen bzw. ob so etwas wie ein ungeschriebenes Tatbestandsmerkmal eingeschoben werden sollte, um einen klar erkennbaren Rechtsgüterschutz zu erreichen (teleologische Restriktion).

Ein legitimer Zweck des hier zu interpretierenden $\S 232$ Abs. 1 StGB n.F. wäre der Schutz der sozialen und wirtschaftlichen Bewegungsfreiheit von Prostituierten vor Ausbeutung. Im Gegensatz zum Straftatbestand der sexuellen Nötigung oder dem schweren Menschenhandel (dort werden auch Lagen erfasst, die als Zwang und Täuschung mit herkömmlichen strafrechtlichen Kategorien gefasst werden können), greifen Straftatbestände, welche ausschließlich strukturelle Zwangslagen berücksichtigen wollen, erheblich weiter.

Umso schlimmer, dass das Wort "Ausbeutung « in unterschiedlichen Gesetzestexten 
höchst uneinheitlich verwendet (von der Rechtsprechung aber einheitlich eng ausgelegt) wird. Zum einen umschreibt § 232 Abs. 1 StGB n.F. einzelne sexuelle Handlungen, zu denen potentielle Opfer gebracht werden, mit diesem Zusatz, nicht aber die Prostitution als solche. Das legt zumindest sprachlich nahe, den Relativsatz: »durch den sie ausgebeutet wird «, nur auf die nach einem »oder " aufgeführten sexuellen Handlungen zu beziehen. Dann bleibt die erste Variante des § 232 Abs. 1 StGB aber so uferlos weit, wie wir dies eingangs unterstellt haben. Noch weiter ist die 2. Alternative, die Jugendschutznorm, da hier unter 21 Jahre alte potentielle Opfer allein ihres Alters wegen geschützt werden sollen ${ }^{5}$. Eine nur vordergründig politisch korrekte weite Auslegung des neu geschaffenen $\S 232$ Abs. 1 StGB würde also zum einen dazu führen, dass schon etwa der "gut gemeinte Rat" eine wirtschaftliche Zwangslage mittels Aufnahme der Prostitution abzumildern, dem Tatbestand des § 232 Abs. 1 S. 1 Alt. 1 StGB unterfiele und zum anderen, dass das 2002 geschaffene Prostitutionsgesetz durch die Jugendschutznorm in S. 2 nicht nur de de facto, sondern de jure auf Menschen über 21 Jahre begrenzt würde. § 232 StGB würde - weit ausgelegt - wie ein allgemeines Verbotsgesetz für alle in diesem Gewerbe tätigen Vermittler und Bordellbetreiber wirken und nachträglich den 2002 hergestellten Kompromiss zurückschrauben.

Die seit 1993 bestehenden und 2005 geänderten und teilweise ganz neu gefassten strafrechtlichen Regelungen des Menschen- und Frauenhandels sind zumindest in Teilen weder einleuchtend noch praktikabel und verbessern auch nicht die Lage der Menschen, die zu Niedriglöhnen im sexuellen Dienstleistungssektor arbeiten.

Ein Strafgericht muss also genau überlegen, ob die Gesetzgebung so weitgehende Folgen bedacht hat und wird dabei erkennen, dass die Beratungen insoweit höchst unergiebig sind. Es dominieren Schilderungen, welche eigentlich nur das Phänomen der »Zwangsprostitution « umschreiben, nicht aber das ganz normale grenzüberschreitende Vermittlungsgeschäft. Offenbar wollte man nicht nur - so unbemerkt wie nur möglich - das Prostitutionsgesetz einschränken, sondern extreme Missstände angehen. Dann aber müsste man den sprachlich uferlosen und konturlosen Tatbestand einschränkend auslegen. Verlangt werden müsste für die unter 21-jährigen, dass auch bei ihnen eine wirtschaftliche Zwangslage ausgenutzt wird und für alle Tatvarianten wäre zu verlangen, dass es sich der Sache nach um Vermittlungstätigkeiten für eine ausbeuterische Prostitution im Sinne des $§ 180$ a StGB handelt, um Wuchergeschäfte also. Strukturelle Probleme können nun einmal nicht strafrechtlich geregelt werden. Hier kann man nur arbeitrechtliche Regelungen auf EU-Level anstreben. Zwar wird dann der deutsche Markt unter Druck geraten, da die Nachfrage für osteuropäische Sex-Arbeiterinnen riesig ist - aus Gründen, die wir hier nicht darlegen können, aber eines erscheint uns klar zu sein: wegen der großen Nachfrage von einer gigantischen Organisierten Kriminalität osteuropäischer Banden zu reden, ist jedenfalls eine Verschiebung und auch eine Übertreibung, bei der sich interessanterweise aber ganz unterschiedliche Lobbyisten beteiligen ${ }^{6}$. Märkte kann man nicht durch Strafrecht regulieren, dies zeigen die $\$ \S 232-233$ a StGB mehr als deutlich. Wenn man strafrechtlich intervenieren will, dann muss man sich auf Phänomene beschränken, die nachprüfbar den Namen »Zwangsprostitution « verdienen. Illegitim ist es jedenfalls, der Mehrzahl der Migrantinnen abzusprechen, dass sie zumindest im Ansatz eine rationale Entscheidung getroffen haben, wenn sie sich für einen solchen riskanten Lebensweg entschieden haben ${ }^{7}$. Die Verwirklichungschancen für eine Kontrolle der alten und neu entstandenen Märkte würden jedenfalls steigen, wenn wir zumindest in Ansätzen ein EU-Arbeitsrecht für sexuell Dienstleistungen hätten.

\section{Fußnoten:}

1 vgl. hierzu auch Thoma in diesem Heft. Die Rechtsprechung betrifft meist die früher von der Polizei noch als verboten angesehene dirigistische Zuhälterei und ist insoweit seit 2002 mehr oder weniger überholt, weil das Dirigieren von Prostituierten Teil des eingeschränkten Direktionsrechts ist. Umso wichtiger wäre eine angemessene Auslegung des Begriffs »Ausbeutung $« . \mathrm{Zu}$ restriktiv ist die Definition "Haltens in sozialer und wirtschaftlicher Abhängigkeit nach BGH NStZ -RR 2003, 361. Nicht gesehen wird ferner im Schrifttum, dass bestimmte Teilaspekte, welche Prostituierten typischerweise das Leben schwer machen, eigentlich bereits als verbotenes Wuchergeschäft nach § 291 StGB zu beurteilen wären. Die Auslegung müsste sich also an diesem Straftatbestand orientieren. Auch Margarete von $\mathrm{Ga}$ len, Rechtsfragen der Prostitution, 2004, Rdnr. 367-368 m.w.Nachw. ist hier wenig weiterführend, da sie in erster Linie die entkriminalisierende Funktion des ProstG betont und seine Gestaltungskraft auf die Möglichkeit faktischer Arbeitsverträge be- schränkt. Aber welchen Schutz Selbstständige benötigen, bleibt offen. Noch unbefriedigender ist die m.E. viel zu kurz greifende Zusammenfassung der Wirkungen des ProstG aus polizeilicher Sicht von Schmidbauer, NJW 2005, $871 \mathrm{ff}$. Beklagt werden Kontrolldefizite, das ProstG als solches wird attackiert, die üblichen Forderungen zu neuen polizeilichen Befugnisnormen wiederholt statt die Probleme der Praxis mit dem Markt sexueller Dienstleitungen $\mathrm{zu}$ benennen.

2 Ein Instrument, das in Zeiten der erweiterten EU zunehmend stumpf wird in den Augen der polizeilichen Beobachter.

3 Kinzig, Die rechtliche Bewältigung von Erscheinungsformen organisierter Kriminalität, Untersuchungen und Forschungsberichte des MPI Freiburg 17, 2004.

4 Exemplarisch hierfür sei nur auf den Bestrebungen der CDU/CSU-Mehrheit im Bundesrat hingewiesen. Im Gesetzgebungsverfahren zielten diese darauf $\mathrm{ab}$ die mit dem ProstG aufgehobene Strafbarkeit der Förderung der Prostitution wiederherzustellen, da die Behörden der Strafverfolgung nun keine zureichenden Ermittlungsansätze mehr hätten, um in das Rotlichtmilieu einzudringen (BT-Drs. 15/4380 S. 2). Noch deutlicher formuliert wird dieses Anliegen im bayerischen Antrag vom 24.02.2005 zur Einführung einer Freierbestrafung. In dieselbe Richtung gehen Äußerungen insb. von Vertretern der Polizei, welche als Reaktion auf das ProstG den Einsatz und Ausbau insb. der präventiven Telekommunikationsüberwachung vorschlagen, vgl. nur Schmidbauer, NJW 2005, S. 871ff.

5 Denn auf das Ausnutzen einer Zwangslage wurde in der Jugendschutznorm des $\S 232$ Abs. 1 S. 2 StGB verzichtet. Sie beseitigt die Geltung des ProstG für Heranwachsende.

6 Etwas vorsichtiger, aber im Ergebnis ebenso Elvira Niesner, Frauenhandel zwischen Tabuisierung, Dramatisierung und Instrumentalisierung - Herausforderung für die feministische Forschung und Praxis durch ein internationales und tagespolitisches Problem, in: Hornung, Gümen, Weilandt (Hg,), Zwischen Emanzipationsvision und Gesellschaftskritik (2001) S. 238, 250ff., die darauf hinweißt, wie etwa bei der Homogenisierung der Opfer von Menschen - und Frauenhandel GO's und NGO's zwar verschiedene Ziele, aber ähnliche Strategien verfolgen.

7 Vgl. Frommel in diesem Heft. Das BKA geht davon aus, dass 32,3 Prozent der Frauen bei der Anwerbung im Heimatland mit der Ausübung Prostitution im Zielland einverstanden waren, 23,5 Prozent der Frauen gingen bereits im Heimatland der Prostitution nach (Lagebild Menschenhandel 2003, S. 11f.); teilweise wird die »Freiwilligenquote $s$ sehr viel höher, nämlich mit 40-50 Prozent angesetzt (Otholt, Prostitution - Frauenhandel - eine eindeutige Verbindung?, in: Gehl (Hg.), Die Ware Mensch, Menschenhandel als Geschäftfeld der Organisierten Kriminalität (2004), S. 21, 24).

Martin Schaar promoviert in Kiel zu diesem Thema, Monika Frommel ist Mitherausgeberin dieser Zeitschrift. 